\title{
Bacterial blood stream infections and antibiogram among febrile patients at Bahir Dar Regional Health Research Laboratory Center, Ethiopia
}

\author{
Derese Hailu, Bayeh Abera ${ }^{2 *}$, Gashaw Yitayew ${ }^{1}$, Daniel Mekonnen² and Awoke Derbie ${ }^{2}$ \\ ${ }^{1}$ Bahir Dar Regional Health Research Laboratory Center \\ ${ }^{2}$ Department of Microbiology, Immunology and Parasitology, College of Medicine and Health Sciences, Bahir Dar \\ University
}

\begin{abstract}
Bacterial blood stream infections (BSIs) are a common cause of morbidity and mortality. Prevailing data on bacterial species causing BSI and their antibiogram are essential for proper management of patients. A retrospective study was conducted on blood culture results that had been processed from March 2013 to January 2015 at Bahir Dar Regional Health Research Laboratory Center. In January 2015, data on age, sex of patients and bacterial isolates with antibiogram were extracted from registration log book. Blood stream causing bacteria were identified according to standard operational procedure for blood culture. Antimicrobial susceptibility tests were performed according to Kirby-Bauer disc diffusion methods. A total of 561 blood specimens were requested for blood culture. Of these, 220 (39.2\%, 95\% CI: 35.3-43.4\%) blood cultures had aerobic bacterial growth. Gram negative bacterial isolates constituted $115(52.3 \%)$ of the isolated bacteria. Staphylococcus aureus $50(22.7 \%)$, coagulase negative staphylococci 35(15.9\%), Klebsiella pneumoniae 35 (15.9\%), Escherichia coli 19 (8.6\%), Pseudomonas aeruginosa 15 (6.8\%) and Acinetobacter species 13(5.9\%) were the most dominant isolates. Overall, drug resistance for gram positive bacteria were 7 to $61 \%$ and for gram negatives 6.9 to $82.6 \%$. Among the gram positive bacteria, high resistance levels were observed against penicillin $(61 \%)$ and oxacillin $(52.9 \%)$. The gram negative bacterial isolates showed 66 to $82.6 \%$ resistance to ampicillin, ceftriaxone and trimethoprim-sulfamethoxazole. The present study revealed that bacterial blood stream infections linked with high levels of drug resistance would pose a challenge in treatment of patients with BSIs. Hence, blood culture with antibiotic susceptibility tests could play key role for appropriate treatment of patients with bacterial blood stream infection.
\end{abstract}

Key words: Blood culture, Bloodstream infection, antibiogram, Ethiopia

DOI: http://dx.doi.org/10.4314/ejst.v9i2.3

\section{INTRODUCTION}

Bloodstream infections (BSIs) cause self-limiting infections to life threatening sepsis and account for significant mortality and morbidity worldwide. Blood stream infection accounts for $10-20 \%$ of all nosocomial infections and is the eighth leading cause of mortality (James et al., 2002). In subSaharan countries including Ethiopia BSI is an important cause of illness and death in children, the mortality rate approaches 53\% which makes it a significant health problem in developing countries (Mehdinejad et al., 2009).
Bloodstream infections are often complicated with syndromes associated with septic shock (Balk, 2000). Bacteria present in circulating blood whether continuously or intermittently are a threat to every organ in the body usually (Vanitha et al., 2012). Individuals with bacteremia may develop septicemia, a life-threatening condition in which multiplying bacteria release toxin in to the blood stream and trigger the release of cytokines, causing fever, chills, malaise and lethargy, with difficulty in breathing especially in children (Shahla et al., 2009; Ehwarieme et al., 2011).

\footnotetext{
*Corresponding author: bayeabera15@gmail.com

(C) This is an Open Access article distributed under the terms of the Creative Commons Attribution License (http://creativecommons.org/licenses/CC BY4.0).
} 
Development of resistance to antimicrobial agents further adds complication to its proper treatment outcome. Studies from Ethiopia and worldwide have reported an increased antimicrobial resistance among bacterial isolates causing BSIs (Usha et al., 2007; Kaistha et al., 2009; Kingsley et al., 2013). In different studies a wide range of organisms have been isolated in BSIs such as Acinetobacter species, Pseudomonas aeruginosa, Escherichia coli, Klebsiella pneumoniae and Neisseria meningitidis. Furthermore, gram positive bacteria such as coagulase negative staphylococci (CoNS), Staphylococcus aureus, enterococci, and alphahemolytic streptococci (Atul et al., 2007; Anu et al., 2010 Kavitha et al., 2010).

Blood culture in order to isolate pathogens and determine drug sensitivity of the isolates remains the main stay of definitive diagnosis and management of BSIs (Shahla et al., 2009). Early identification of bacteria causing BSI and their antimicrobial susceptibility is essential for rapid administration of antimicrobial therapy to patients with BSIs. This has shown to improve treatment outcomes (Munson et al., 2003).

In Ethiopia, a few studies documented data on bacterial blood stream infections and antimicrobial resistance profiles of blood stream causing bacterial species (Tizazu Zenebe et al., 2011; Mulat Dagnew et al., 2013; Araya Gebreyesus et al., 2015). However, data on bacterial blood stream infections are not documented in the study area. Therefore, this study was conducted to determine the bacterial gents and antimicrobial susceptibility profiles of blood stream infections among patients referred to Bahir Dar Regional Health Research Laboratory Center, North West Ethiopia.
METHODS

\section{Study settings}

A retrospective study was conducted on blood culture results that had been processed from March 2013 to January 2015 at the Bahir Dar Regional Health Research Laboratory Center. In January 2015, data on age, sex of patients and bacterial isolates with their antibiogram were extracted from laboratory registration records of using a standard data extraction sheet. Bahir Dar Regional Health Research Laboratory Center is the technical arm of Amhara Regional Health Bureau currently providing culture and susceptibility tests, MDRTB culture and molecular laboratory techniques like real time PCR and quality assurance services to Felege Hiwot referral Hospital, nearby health centers, private hospitals and clinics.

\section{Culture and identification}

According to the standard operational procedures for blood culture, $5 \mathrm{ml}$ and $2 \mathrm{ml}$ blood samples were aseptically collected from adults and children, respectively. The vein puncture sites were cleaned with $70 \%$ alcohol and dried before collection of blood sample. Collected blood samples were inoculated onto Tryptic Soy Broth (Oxoid, UK) in $1: 5$ to $1: 10$ proportions and incubated at $37^{\circ} \mathrm{C}$. Overnight incubated culture bottles were subcultured onto sheep blood agar, chocolate blood agar and MacConkey agar. Negative blood culture results would be followed till fifth days up by examining the daily before issued results.

Preliminary identification of the isolates was made on agar based on macroscopic colony characteristics. Gram-negative rods were identified by series of biochemical tests such as Indole, Simon's citrate, 
Urea, Triple sugar iron (TSI), lysine iron, and motility. Gram-positives were identified based on their preference of growth on blood agar plate followed by catalase, coagulase, bacitracin and optochin tests (Wikler et al., 2007).

\section{Antimicrobial susceptibility testing}

The antimicrobial susceptibility testing was performed according to Kirby-Bauer disk diffusion method on Mueller Hinton agar plates (Oxoid, UK) (Wikler et al., 2007). The antimicrobials tested were obtained from Oxoid Ltd. UK with the following concentrations: ampicillin $(10 \mu \mathrm{g})$, amikacin $(30 \mu \mathrm{g})$, ciprofloxacin $(5 \mu \mathrm{g})$, trimethoprim/ sulphamethoxazole $1.25 / 23.75 \mu \mathrm{g})$, pepracillin $(100 \mu \mathrm{g})$, gentamicin $(10 \mu \mathrm{g})$, tetracycline $(30 \mu \mathrm{g})$, vancomycin $(30 \mu \mathrm{g})$, penicillin (10IU), clindamycin $(30 \mu \mathrm{g})$, ceftriaxone $(30 \mu \mathrm{g})$, chloramphenicol $(30 \mu \mathrm{g})$, ceftazidime $(30 \mu \mathrm{g})$, and oxacillin $(30 \mu \mathrm{g})$. Grades of susceptibility were determined after incubation at $35^{\circ} \mathrm{C}$ for 24 hours according to Clinical Laboratory Standards Institute (CLSI) (Wikler et al., 2007).

\section{Quality control}

The standard reference strains S. aureus (ATCC 25923), E. coli (ATCC 25922) and P. aeruginosa (ATCC 27853) were used to check the potency of antimicrobial discs, to control drug susceptibility testing procedures and for identification of bacterial species. The absence of bacterial growth in un inoculated media was done by randomly taking the prepared culture media and incubating over nigh to see for any growth.

\section{Data analysis}

Data were analyzed using Statistical Package for Social Sciences version 20 Software (IBM Corp. Released 2011. IBM SPSS Statistics for Windows,
Version 20.0. Armonk, NY: IBM Corp). The chisquare test was employed to compare the proportion of bacterial isolates with patients' age and sex and $p$-value of less than 0.05 was considered as statistical significance.

\section{Ethical statements}

Ethical clearance was obtained from Amhara Regional Health Bureau Institutional Review Board (IRB) at Bahir Dar Regional Health Research Laboratory Center to get permission for scientific publication of the data.

\section{RESULTS}

From March 2013 to January 2015, a total of 561 blood specimens were requested for culture and antimicrobial susceptibility tests. Among these, 289 $(51.5 \%)$ were from females patients. The median age of patients was 15.5 years with a range of 4 days to 75 years. Overall, 220 (39.2\%) of blood specimens had aerobic bacterial growth. Among aerobic culture positives, 203 (92.3\%) were monomicrobial and 13 (5.9\%) were polymicrobial growth.

Table 1 depicts blood culture results with patient characteristics. Regards to sex, 104 (38.2\%) male patients had blood culture positive while 116 (40.1\%) females had blood culture positive. Statistically significant associations were between age and status of blood culture $(\mathrm{P}=0.001)$. Highest percentage 115 (70.1\%) of microbial isolates were reported in the age group of patients less than one year.

Table 2 demonstrates bacterial species isolated from blood cultures. S. aureus was the predominate isolate with $50(22.7 \%)$ followed by coagulase neg- 
Table 1. The distribution of blood culture results by patients' age and sex (Mar 2013-Jan, 2015)

\begin{tabular}{llcc}
\hline \multirow{2}{*}{ Variables } & \multicolumn{2}{c}{ Blood culture results } & P value \\
\cline { 2 - 4 } Age (Years) & Culture negative N (\%) & Culture positive N (\%) & \\
$<1$ & $49(14.4)$ & $115(70.1)$ & 0.001 \\
$1-12$ & $96(66.7)$ & $48(33.3)$ & \\
$13-18$ & $47(73.4)$ & $17(26.6)$ & \\
$>18$ & $149(78.8)$ & $40(21.2)$ & 0.645 \\
Total & $341(60.8)$ & $220(39.2)$ & \\
Sex & & $104(38.2)$ & \\
Male $(\mathrm{n}=272)$ & $168(61.8)$ & $116(40)$ & \\
Female $(\mathrm{n}=289)$ & $173(60)$ & $220(39.2)$ & \\
Total & $341(60.8)$ &
\end{tabular}

ative staphylococci (CoNS) 35 (15.9\%), K. pneumoniae 35 (15.9\%), E. coli 19 (8.6\%), P. aeruginosa 15 (6.8\%) and A. baumanni 13 (5.9\%). Gram negative bacterial isolates constituted 115 (52.3\%) of all isolates.
The antimicrobial susceptibility profiles of gram positive and gram negative isolates are shown in Table 3 and 4. Among the gram positive bacteria, high resistance level was observed against penicillin $(61 \%)$ and oxacillin $(52.9 \%)$. The gram

Table 2. Frequency of bacterial species isolates from blood culture (Mar 2013-Jan, 2015)

Bacterial Group $\quad$ Number (\%)

\section{Gram positive bacteria}

Staphylococcus aureus

Coagulase negative staphylococci

Eenterococcus faecalis

$6(2.7)$

Others

\section{Gram negative bacteria}

Escherichia coli

Acinetobacter baumanni

Klebsiella pneumoniae

Eenterobacter clonae

Pseudomonas aeruginosa

Proteus mirabilis

Proteus vulgaris 
Table 3. Antimicrobial resistance profiles of gram positive bacteria from blood cultures (Mar 2013-Jan, 2015)

Bacterial species

Antimicrobial resistance N (\%)

\begin{tabular}{|c|c|c|c|c|c|c|c|c|c|}
\hline & OX & CIP & SXT & VA & $\mathrm{TE}$ & $P$ & $\mathrm{C}$ & DA & $\mathrm{CN}$ \\
\hline S. aureus & $33(66)$ & $10(20)$ & $20(48.7)$ & NT & $48(96)$ & $39(78)$ & $25(50)$ & $7(14)$ & NT \\
\hline CoNS & $13(37.1)$ & $3(8.5)$ & $13(37.1)$ & NT & $2(5.7)$ & $17(48.5)$ & $925.7)$ & $3(8.5)$ & NT \\
\hline E. fecalis & NT & NT & NT & $1(16.6)$ & NT & NT & $1(16.6)$ & NT & $1(16.6)$ \\
\hline S. pneumoniae & NT & NT & NT & $0(0)$ & NT & $0(0)$ & NT & NT & NT \\
\hline S. viridians & NT & NT & NT & $0(0)$ & NT & $0(0)$ & NT & $1(25)$ & NT \\
\hline S. pyogenes & NT & $0(0)$ & $0(0)$ & $1(33.3)$ & NT & $2(66.6)$ & $1(33.3)$ & $1(33.3)$ & NT \\
\hline Micrococcus spp. & $0(0)$ & $0(0)$ & NT & NT & NT & $0(0)$ & $0(0)$ & $0(0)$ & NT \\
\hline Total $(\mathrm{n}=101)$ & $46(52.9)$ & $13(14.4)$ & $23(26.1)$ & $2(14.3)$ & $6(7)$ & $58(61)$ & $36(37.5)$ & $12(12.8)$ & $1(16.6)$ \\
\hline
\end{tabular}

Key: $\quad \mathrm{OX}=$ Oxacillin; $\quad \mathrm{CIP}=$ ciprofloxacin; $\quad \mathrm{SXT}=$ Trimethoprim + Sulphamethoxazole; $\quad \mathrm{VA}=$ Vancomycin; $\mathrm{TE}=$ Tetracycline, $\mathrm{P}=$ Penicillin; $\mathrm{C}=\mathrm{Ch}$ loramphenicol; $\mathrm{DA}=$ Clindamycin; $\mathrm{CN}=$ Gentamycin; $\mathrm{NT}=$ Not tested

negative organisms showed $66-82.6 \%$ resistance to ampicillin, ceftriaxone and trimethoprimsulfamethoxazole. The overall the range of resistance in gram positive and negative bacteria are shown in table 4.

\section{DISCUSSION}

Studies show that BSIs are frequently encountered by clinicians mainly in developing countries. BSI is one of the major infections for frequent antibiotic use (Vanitha et al., 2012; Mulat Dagnew et al., 2013). In the present study, blood culture was one of the frequently requested specimens for culture and antimicrobial susceptibility tests. This indicates that BSI is a common health problem of the community which could partly explained by $39 \%$ prevalence of blood stream infection.

The blood culture positivity rate in this study is higher compared to other studies in Ethiopia which reported $18.2 \%$ and $8.8 \%$ positivity rate (Tizazu Zenebe et al., 2011; Mulat Dagnew et al., 2013). The isolation rate of bacteria in the present study is also higher than studies reported in other parts of the world like in South India (8.39\%), Iran $5.6 \%$, Nigeria $19.3 \%$ and $20.02 \%$ and $9.44 \%$ 
Table 4. Antimicrobial resistance profiles of gram negative isolates from blood cultures (Mar 2013-Jan, 2015)

\begin{tabular}{|c|c|c|c|c|c|c|c|c|c|}
\hline \multirow[t]{2}{*}{ Bacterial species } & \multicolumn{9}{|c|}{ Antimicrobial resistance $\mathrm{N}(\%)$} \\
\hline & AMP & $\mathrm{CRO}$ & CIP & SXT & $\mathrm{AK}$ & $\mathrm{CN}$ & $\mathrm{C}$ & PRL & CAZ \\
\hline E. coli & $14(73.7)$ & $12(63.1)$ & $5(26.3)$ & $15(78.9)$ & $0(0)$ & $10(52.6)$ & $3(15.7)$ & NT & NT \\
\hline Acinetobacter spp & $10(76.9)$ & $10(76.9)$ & $2(15.3)$ & $8(61.5)$ & $1(7.6)$ & $7(53.8)$ & $9(69.2)$ & NT & NT \\
\hline K. pneumoniae & $32(91.4)$ & $26(74.2)$ & $10(28.5)$ & $27(77.1)$ & $2(5.7)$ & $25(71)$ & $16(45.7)$ & NT & NT \\
\hline E. clonae & $8(100)$ & $7(87.5)$ & $1(12.5)$ & $4(50)$ & $2(25)$ & $6(75)$ & $6(75)$ & NT & NT \\
\hline P. aeruginosa & $15(100)$ & NT & $2(13.3)$ & NT & $1(6.6)$ & $3(20)$ & NT & $4(26.6)$ & $8(53.3)$ \\
\hline P. mirabilis & $3(60)$ & $3(60)$ & $0(0)$ & $1(20)$ & $0(0)$ & $0(0)$ & $2(40)$ & NT & NT \\
\hline P. vulgaris & $0(0)$ & $0(0)$ & $0(0)$ & $0(0)$ & $0(0)$ & $0(0)$ & $0(0)$ & NT & NT \\
\hline Salmonella spp & $3(60)$ & $3(60)$ & $1(20)$ & $4(80)$ & $0(0)$ & $2(40)$ & $1(20)$ & NT & NT \\
\hline Providencia spp & $2(66.6)$ & $1(33.3)$ & $0(0)$ & $1(33.3)$ & $0(0)$ & $1(33.3)$ & $0(0)$ & NT & NT \\
\hline K. ozenae & $2(50)$ & $2(50)$ & $1(25)$ & $2(50)$ & $0(0)$ & $2(50)$ & $1(25)$ & NT & NT \\
\hline$K$. rhinose & $2(100)$ & $2(100)$ & $1(50)$ & $1(50)$ & $0(0)$ & $1(50)$ & $1(50)$ & NT & NT \\
\hline Citrobacter spp & $4(100)$ & $2(50)$ & $1(25)$ & $3(75)$ & $2(50)$ & $3(75)$ & $3(75)$ & NT & NT \\
\hline Total $(n=115)$ & $95(82.6)$ & $68(68)$ & $24(20.8)$ & $66(66)$ & $8(6.9)$ & $60(52.1)$ & $27(27)$ & $4(26.6)$ & $8(53.3)$ \\
\hline
\end{tabular}

Key: $\mathrm{AMP}=$ Ampecillin; $\mathrm{CRO}=$ Ceftriaxone; $\mathrm{AK}=$ Amikacin; $\mathrm{PRL}=$ Pepracillin; $\mathrm{CAZ}=$ Ceftazidime

in India (Usha et al., 2007; Kalantar et al., 2008; Mehdinejad et al., 2009; Ehwarieme et al., 2011; Vanitha et al., 2012; Sumita et al., 2014). The possible explanation for these differences could be differences in age range of patients, administration of antibiotics before blood culture, laboratory procedure, geographical location, seasonal variation and epidemiological difference of the etiological agent (Pitout and Laupland, 2008).

In the present study, gram negative bacteria were more frequently isolated than gram positives. This 
conforms to a study in Lahore which reported $60 \%$ of gram negative and $40 \%$ of gram positives (Majda et al., 2011). Likewise, in India $52.82 \%$ gram negative and $46.56 \%$ gram positive bacteria were isolated in blood stream infections (Amit et al., 2014). In contrast, $31 \%$ of gram negative and $69 \%$ of gram positive bacteria were reported from Gondar (Mulat Dagnew et al., 2013) and 39.1\% gram negative and $60.9 \%$ gram positive from Jimma, Ethiopia (Tizazu Zenebe et al., 2011). The predominant isolate of this study was S. aureus $(22.7 \%)$ which is comparable with other studies (Vanitha et al., 2012; Kalpesh et al., 2014; Sumita et al., 2014). Coagulase negative staphylococci (CoNS) have been considered as non pathogens believed that it could be skin flora specially when isolated in a single blood culture. But, their role as pathogens was reported in different studies as an important cause of morbidity and mortality in immune suppressed individuals (Usha et al., 2007; Shahla et al., 2009; Tizazu Zenebe et al., 2011).

The rate of antibiotic resistance in this study is comparatively higher than previous reports in Ethiopia (Tizazu Zenebe et al., 2011; Mulat Dagnew et al., 2013) and other studies conducted elsewhere in the world (James et al., 2002; Amit et al., 2014). A high prevalence of antibiotic resistance was noticed in this study especially among gram negative bacteria. This might be due to none judicious use of antibiotic in health facilities and easy availability of drugs in the market (Bayeh Abera et al., 2014).

Amongst the gram positive bacteria, high resistance was observed to penicillin (61\%) and oxacillin $(52.9 \%)$ which is almost consistent with a study by Kalantar et al. (2008) that recorded about $41 \%$ resistance to oxacillin and $60 \%$ for penicillin. Furthermore, high resistance among gram negative was seen against ampicillin (82\%), ceftriaxone
(68\%) and thrimetoprim-sulfamethoxazole (66\%) which is concordant with the study finding by Mehdinejad et al. (2009).

Among gram-positive bacteria, S. aures showed high resistance level (48-96\%) against trimetoprim-sulphamethoxazole, oxacillin, penicillin and tetracycline which are commonly prescribed antimicrobial agents in the area (Bayeh Abera, 2014). This result was similar with a report in other studies in Ethiopia (Tizazu Zenebe et al., 2011; Araya Gebreyesus et al., 2015) and in Nigeria (Ehwarieme et al., 2011). However, clindamycin and ciprofloxacin were effective against $S$. aureus. In Ethiopia, uncontrolled over the counter sale of antimicrobial agents, mainly for self treatment without health professionals prescription would inevitably lead to emergence and rapid dissemination of antibiotic resistance in the community (Bayeh Abera et al., 2014).

\section{CONCLUSION}

This study showed that bacterial blood stream infections are common in all age groups of febrile patients. However, bacterial blood culture positivity rate was higher in febrile children below 1 year than other age groups. Overall, bacterial isolates in blood culture showed high levels of antibiotic resistance against commonly prescribed antimicrobials. Hence, blood culture with antibiotic susceptibility tests could play key role for appropriate treatment of patients with blood stream infection.

\section{ACKNOWLEDGMENTS}

The authors would like to greatly acknowledge the staff members of department of microbiology at Bahir Dar Regional Health Research Laboratory Center for their valuable professional help during data collection. 


\section{REFERENCES}

Amit, K.S., Vimala, V., Ravinder, P.S and Mastan, S. (2014). Bacterial and antimicrobial resistance profile of bloodstream infections: A hospitalbased study. Chrismed Journal of Health and Research 1:140-144.

Anu, G., Shweta, S., Anita, A and Ashish, G. (2010). Changing trends of in vitro antimicrobial resistance Patterns in blood isolates in a tertiary care hospital over a period of 4 years. Indian Journal Medical Sciences 64: 485-492.

Araya Gebreyesus Wasihun, Letemichael Negash Wlekidan, Senay Aregawi Gebremariam,Tsehaye Asmelash Dejene, Abadi Luel Welderufael, Tadesse Dejenie Haile and Saravanan M. (2015). Bacteriological profile and antimicrobial susceptibility patterns of blood culture isolates among febrile patients in Mekelle Hospital, Northern Ethiopia. Springer Plus 4:314 DOI 10.1186/ s40064-015-1056-x.

Atul, G., Anupurba, S., Jaya, G., Goyal, R.K and Sen, M.R. (2007). Bacteriological Profile and Antimicrobial Resistance of Blood Culture Isolates from a University Hospital. Journal, Indian Academy of Clinical Medicine 8:13943.

Balk, R.A. (2000). Severe sepsis and septic shock. Definitions, epidemiology and clinical manifestations. Critical Care Clinics 16:179192.

Bayeh Abera, Mulugeta Kibret and Wondemagegn Mulu. (2014). Knowledge and beliefs on antimicrobial resistance among physicians and nurses in hospitals in Amhara Region,
Ethiopia. BMC Pharmacology and Toxicology 15:26.

Ehwarieme, D.A., Egbule, O.S and Omonigho. O. (2011). Study of prevalence and antimicrobial susceptibility of blood culture bacterial isolates. Malaysian Journal of Microbiology 7: 78-82.

James, A.K., Mark, E.J, Deborah, C.D, Clyde, T., Daniel, F.S and Gregory, A.V. (2002). Prevalence and antimicrobial susceptibilities of bacteria isolated from blood cultures of hospitalized patients in the United States in 2002. Annals of Clinical Microbiology and Antimicrobials http://www.ann-clinmicrob. com/content/3/1/7. Accessed 14 Feb 2015.

Kaistha, N., Mehta, M., Singla, N., Garg, R., Chander, J. (2009). Neonatal septicemia isolates and resistance patterns in a tertiary care hospital of North India. Journal of Infection in Developing countries 4:5557.

Kalantar ,E., Motlagh, M., Lordnejad, H and Beiranvand, S. (2008). The prevalence of bacteria isolated from blood cultures of Iranian children and study of their antimicrobial susceptibilities. Jundishapur Journal of Natural Pharmaceutical Products 3(1): 1-7.

Kalpesh, G., Amit, J., Shailesh, S., Sishir, G., Ravindra, Sand Mahesh, D. (2014). Bacteriological Profile and Drug Resistance Patterns of Blood Culture Isolates in a Tertiary Care Nephrourology Teaching Institute. Hindawi BioMed Research International http://dx.doi.org. Accessed 15 Feb 2015.

Kavitha, P., Sevitha, B and Sunil, R. (2010). Bacteriologic profile and antibiogram of blood culture isolates in a pediatric care 
unit. Laboratory Physicians 2: 85-88.

Kingsley, O.C., Ifeanyi, A.O, Edet, A.E and Smart, O.C. (2013). Bacteriologic profile and antibiotics susceptibility pattern suspected septicemic patients in Uyo, Nigeria. Research Journal of Medical science 7: 35-39.

Majda, Q and Farooq, A. (2011). Prevalence of microbial isolates in blood cultures and their antimicrobial susceptibility profiles. Biomedical 27:136-139.

Mehdinejad, M., Khosravi, A.D and Morvaridi, A. (2009). Study of prevalence and antimicrobial susceptibility pattern of bacteria isolated from blood cultures. Journal of Biological Sciences 9: $249-253$.

Mulat Dagnew, Gizachew Yismaw, Mucheye Gizachew, Alemayehu Gadisa, Tigist Abebe, TinebebTadesse, Agersew Alemu and Biniam Mathewos. (2013). Bacterial profile and antimicrobial susceptibility pattern in septicemia suspected patients attending Gondar University Hospital, Northwest Ethiopia. BMC Research Notes 6 (283):1-7.

Munson, E.L., Diekema, D.J., Beekmann, S.E, Chapin, K.C and Doern, G. (2003). Detection and treatment of bloodstream infection: Laboratory reporting and antimicrobial management. Journal of Clinical Microbiology 41:495497.

Pitout, J. D and Laupland, KB. (2008). Extendedspectrum beta-lactamase-producing Enterobacteriaceae: an emerging publichealth concern. Lancet Infectious Diseases 8:159-166.

Shahla. L., Anwar, M.S and Ishtiaq, A. (2009).
Bacterial pathogens responsible for blood stream infection (BSI) and pattern of drug resistance in a tertiary care hospital of Lahore. Biomedical 25:101-105.

Sumita, R., Syed, M and Jasmin, P. (2014). Study of prevalence and antimicrobial susceptibility pattern in blood isolates from a tertiary care hospital in North Kerala, India. International Journal of Current Microbiology and Applied Sciences 3: 655-662.

Tizazu Zenebe, Subbaram Kannan, Daniel Yilma and Getenet Beyene. (2011). Invasive bacterial pathogens and their antibiotic susceptibility patterns in Jimma University specialized hospital, Jimma, southwest Ethiopia. Ethiopian Journal of Health Sciences 21:1-7.

Usha, A and Pushpa, D. (2007). Bacterial Profile of Blood Stream Infections and Antibiotic Resistance Pattern of Isolates. JK Sciences 9:186-189.

Vanitha, R.N., Kannan, G., Venkata, N.M., Vishwakanth, D., Nagesh, V., Yogitha, M., Venkata, S.M and Palani, T. (2012). A retrospective study on blood stream infections and antibiotic susceptibility patterns in a tertiary care teaching hospital. International Journal of Pharmacy and Pharmaceutical Sciences 4: 543-548.

Wikler, M.A., Cockerill, F.R., Craig, W.A., Dudley, M.N., Eliopoulo, G.M and Hecht, D.W. (2007). Performance standards for antimicrobial susceptibility testing; seventeenth informational supplement. Clinical Laboratory Standard Institute (M100-S17) 27:25.146. 